International Journal of Modern Physics A, (C) World Scientific Publishing Company

\title{
LOW DIRAC EIGENMODES AND THE TOPOLOGICAL AND CHIRAL STRUCTURE OF THE QCD VACUUM*
}

\author{
H. B. THACKER \\ Department of Physics, University of Virginia
}

Several lattice calculations which probe the chiral and topological structure of QCD are discussed. The results focus attention on the low-lying eigenmodes of the Dirac operator in typical gauge field configurations.

\section{Eigenmode expansion of quark propagators}

Standard methods in lattice QCD consist of calculating various hadronic amplitudes by tying together appropriate combinations of quark propagators calculated on an ensemble of Monte Carlo generated gauge fields. A number of recent lattice QCD calculations have focused increasing attention on the role of low-lying eigenmodes of the Dirac operator in typical background gauge fields. The decomposition of quark propagators into a sum over eigenmodes provides some very useful insight into the structure of low-enery QCD, particularly in exposing connections between quark propagation and chiral dynamics.

\section{Lattice Results}

I will breifly describe several recent and ongoing investigations which employ lattice calculations to address issues of chiral symmetry, gauge topology, and the structure and implications of low Dirac eigenmodes. All of these are discussed in more detail in the cited references.

\subsection{Exceptional configurations and pole-shifting}

The cause of the "exceptional configuration" problem for quenched QCD calculations at light quark mass has been found 1 It arises from the presence of a small number of exactly real eigenmodes which should be at zero quark mass (critical hopping parameter) but which, on some configurations, have migrated to positive quark mass due to the lattice chiral symmetry breaking of the Wilson mass term. The cure consists of shifting these positive mass eigenmodes to zero mass where they belong. Meson and hairpin correlators are then calculated with good statistics down to pion masses of $200 \mathrm{MeV}$ or less.

\subsection{The $\eta^{\prime}$ mass}

\footnotetext{
*Talk presented at the DPF2000 Conference .
} 
Using improved "pole-shifted" quark propagators, the pseudoscalar hairpin (looploop) correlator has been calculated for quark masses ranging from approximately the strange quark mass down to very light quark mass, corresponding to a pion mass less than $200 \mathrm{MeV}$ The time-dependence of the quenched hairpin correlator is very well described by the double-Goldstone pole form expected from a chiral Lagrangian description in which the $q \bar{q}$ annihilation-creation process is treated as an $\eta^{\prime}$ mass insertion. At $\beta=5.7$, the anomaly contribution to the $\eta^{\prime}$ mass is $m_{0}=464(18)$ for unimproved Wilson quarks, and $685(32) \mathrm{MeV}$ for clover-improved quarks, using $C_{s w}=1.57$, and a lattice spacing of $a^{-1}=1.18 \mathrm{GeV}$.

\subsection{Topological susceptibility of quenched QCD}

The integrated anomaly or fermionic method for determining topological susceptibility, originally suggested by Smit and Vink, has been applied to the same ensembles of quenched gauge configurations used to study the $\eta^{\prime}$ mass. ber $\nu$ of each configuration is determined by integrating the pseudoscalar charge and using the integrated axial anomaly, $\int \bar{\psi} \gamma^{5} \psi d^{4} x=i \nu / m$. The result obtained (with clover improved fermions) for the topological susceptibility is $\chi_{t}=(188(4) \mathrm{MeV})^{4}$. This is in good agreement with a phenomelogical estimate based on the $\eta^{\prime}$ mass and the Witten-Veneziano formula.

\subsection{Quenched chiral logarithms}

Anomalous chiral effects of the quenched $\eta^{\prime}$ propagator, originally discussed by Sharpe, Bernard, and Golterman 3 have been confirmed by lattice results. predicted anomalous power law behavior of both the pion mass as a function of quark mass and of the pseudoscalar decay constant as a function of $m_{\pi}^{2}$ are observed. Also as predicted, the axial vector decay constant is found to have smooth analytic behavior in the chiral limit. All the observed quenched chiral log effects are consistent with a value of the QCL parameter of $\delta=.065(13)$ for clover improved quarks.

\subsection{Ghost states in the scalar valence propagator}

A study of the scalar, isovector meson propagator in quenched QCD reveals a strik-

ing behavior which is another QCL effect of the quenched $\eta^{\prime}$ propagator. As the quark mass becomes light, the scalar meson propagator develops a long-range tail which falls off rougly like $\exp \left(-2 m_{\pi} t\right)$ and has the wrong sign, i.e. the long range tail has a sign opposite to that required by spectral positivity. The results agree in detail with the assumption that this tail represents the contribution of a chiral loop diagram containing an $\eta^{\prime}-\pi$ intermediate state, which is light and effectively of negative spectral weight in the quenched theory. In quenched chiral perturbation theory, 3 the tail arises from a ghost fermion-antifermion loop.

\subsection{The truncated determinant algorithm}


The truncated determinant algorithm (TDA) is an approach to full QCD simulations that begins by splitting the closed quark-loop determinant into a product over low Dirac eigenvalues times a product over high eigenvalues $\Delta=\Delta_{l} \Delta_{h}$. The separation is typically made at about 600 to $800 \mathrm{MeV}$. The low end of the determinant $\Delta_{l}$ is treated exactly, using the Lanczos algorthm combined with a Metropolis accept-reject step. The high end of the determinant $\Delta_{h}$ is found to be well approximated by a set of gauge loops, which can be easily included in the Monte Carlo heat bath.

\subsection{The origin of the OZI rule}

Using methods similar to those used to study the $\eta^{\prime}$ hairpin correlator, $q \bar{g}$ annihilation-creation processes in other spin-parity channels have been studied 6 The results confirm the strong OZI suppression in the vector and axial vector channels expected from phenomenology. OZI violation in the scalar channel is found to be large and comparable to the pseudoscalar channel, as predicted from quark model arguments by Isgur and Geiger. a statement about the spin-parity structure of low-lying Dirac eigenmodes.

\subsection{Evidence against instantons in $Q C D$}

Arguments by Witten that topological charge fluctuations in the QCD vacuum come not in the form of instantons but rather from the generically large gauge fluctuations of a confining vacuum are strongly supported by a study of the chiral structure of low Dirac eigenmodes

\section{Acknowledgements}

I am grateful to my collaborators W. Bardeen, A. Duncan, E. Eichten, I. Horvath, N. Isgur, and J. McCune. This work was supported in part by the U. S. Department of Energy under grant DE-FG02-97ER41027.

\section{References}

1. W. Bardeen, A. Duncan, E. Eichten, G. Hockney and H. Thacker, Phys. Rev. D57 (1998) 1633.

2. W. Bardeen, A. Duncan, E. Eichten, and H. Thacker FERMILAB-PUB-00-154-T, heplat/0007010.

3. C. Bernard and M. Golterman, Phys. Rev. D46 (1992) 853; S. Sharpe, Phys. Rev. D46 (1992) 3146.

4. W. Bardeen, A. Duncan, E. Eichten, N. Isgur, and H. Thacker (in preparation).

5. A. Duncan, E. Eichten, and H. Thacker, Phys. Rev. D59 (1999) 014505.

6. N. Isgur and H. Thacker, JLAB-THY-00-10, hep-lat/0005006.

7. P. Geiger and N. Isgur, Phys. Rev. D47, 5050 (1993).

8. I. Horvath, N. Isgur, J. W. McCune, and H. Thacker (in preparation). 